\title{
MULTIPOLE-INDUCED DIPOLE CONTRIBUTIONS TO THE FAR-INFRARED SPECTRA OF DIATOMIC MOLECULES IN NON-POLAR SOLVENTS
}

\author{
A. MEDINA \\ ETSII de Béjar, Universidad de Salamanca, \\ 37700 Béjar, Salamanca, Spain \\ AND \\ J.M.M. ROCO, A. CALVO HERNÁNDEZ AND S. VELASCO \\ Departamento de Física Aplicada, Facultad de Ciencias \\ Universidad de Salamanca, 37008 Salamanca, Spain
}

\begin{abstract}
In this paper we overview our work on far-infrared spectroscopy of both hetero- and homonuclear diatomic molecules in nonpolar fluids. Special attention is paid to electric multipolar induced contributions. From comparison between theoretical and experimental spectra estimation of the leading multipoles of $\mathrm{CO}$ and $\mathrm{N}_{2}$ molecules has been obtained. Also a temperature and density dependence analysis of many-body cancellation effects in the different pure induced components of the far-infrared integrated absorption coefficient of $\mathrm{CO}$ in liquid $\mathrm{Ar}$ is reported.
\end{abstract}

\section{Introduction}

Dilute solutions of diatomic molecules in dense nonpolar, particularly monatomic, solvents form one of the simplest classes of high-density molecular systems which attracted great deal both of theoretical and experimental spectroscopic work during past decades [1]. Some features that make these systems specially challenging are: 1) interactions among solute molecules can be disregarded and their mutual spatial correlations neglected; 2) farinfrared (FIR) and near-FIR experimental spectra show usually well separated pure rotational and vibration-rotational bands free of many complications, such as the Coriolis coupling, Fermi and Darlin-Dennison resonances, anharmonic splittings, etc., arising in the spectra of polyatomics; 
3) nonpolar fluids cause the smallest perturbations to the intramolecular dynamics of solute substances and present a very low absorption over a broad frequency range; 4) absorption spectra give information on molecular constants, as well as on the mechanisms to which absorption can be ascribed; 5) the changes in such spectra upon variation of the bulk parameters (density, temperature, composition) of the system provide information on intermolecular interactions and on their influence on the dynamics of molecular motions.

Far-infrared (FIR) absorption spectra of heteronuclear diatomic molecules dissolved in dense nonpolar solvents have two main contributions. The first one is due to the permanent dipole moment (permanent contribution) of the diatomic itself. The second one comes from the induced dipole moment (induced contribution) of the solution which appears from the interaction between unlike molecules. The relative importance of these contributions depends mainly on the value of the permanent dipole moment of the diatomic and on the density of the fluid. If the permanent dipole moment of the diatomic is high enough, the permanent contribution dominates the FIR absorption spectrum even at liquid densities [2]. However, for low enough permanent dipole moments this term will not be overwhelming and induction absorption mechanisms must be considered, especially for high densities [3,4]. Moreover, in the case of homonuclear diatomics dissolved in rare-gas fluids there is no permanent contribution so the whole FIR absorption spectrum arises from induced contributions [5, 6].

One of our aims in the last years was to theoretically analyze this kind of interaction-induced effects. This analysis has three fundamental problems [1]:

i) The coupling between optically active intramolecular and intermolecular degrees of freedom. In these spectra the relevant degrees of freedom contributing to absorption are the rotation of the diatomic and the translation of the solvent atoms and the diatomic itself. These degrees of freedom are coupled via the anisotropic part of the solute-solvent interaction, but if it is assumed that they are uncorrelated (a usual hypothesis in most spectral theories) the spectra can be expressed as a convolution of a translational part and a rotational one.

ii) The difficulty to determine which induction mechanisms are relevant in each particular system. Interactions among molecules cause shifts in their charge distributions that provoke induced dipole moments depending on the intermolecular separation, and vary as the molecules rotate and vibrate. These interaction-induced dipoles are responsible for several kinds of spectra (in particular FIR absorption) of symmetric non-polar species and contribute to allowed spectra of dipolar molecules [7]. In fact, the interaction-induced component is present in the spectrum of any dense 
molecular system, although it can be vanishingly small compared to the permanent component. The leading long-range mechanism for the dipole moment induction in molecular systems has its origin in the linear polarization of the solvent molecules by the field due to electric multipoles of the diatomic probe. Besides this direct induction, other contributions exist due to back induction (i.e., the dipole moment induced on the solvent polarizes the solute), due to the field-gradient effects, and due to hyperpolarizabilities of the solvent molecules. Ordinarily these are second-order effects at best. Possible though apparently unexplored exceptions could be expected for the systems with resonantly enhanced polarizabilities including usually neglected vibrational polarizability [8].

iii) The usual difficulties arising in all theoretical studies involving manybody effects. A significant intensity decrease with increasing solvent density have been observed in the integrated absorption coefficient (IAC) of both hetero- and homonuclear diatomic molecules in spherical solvents. This decreasing has been attributed to the existence of static cancellation effects between two- and three-body components of the pure induced dipole spherical components. The extent of these cancellation effects represents a measure of the isotropy of the environment around a molecule in the fluid. Furthermore, these cancellation effects decrease when going to high multipolar induction mechanisms, and even for hexadecapole-induced dipole cancellation disappears and transforms into enhancement effects at least for some particular thermodynamical conditions. The nature of these effects remains still not well understood. A significant part of our work in the last years has been devoted to analyze how the translational and rotational degrees of freedom of the system affect the existence of cancellation or enhancement effects $[9,10]$.

This work briefly compiles our main contributions to the study of electric multipole induced contributions to the FIR spectra of diatomic molecules in nonpolar solvents.

\section{Dipole absorption coefficient}

We deal with a diluted solution of diatomic molecules in a nonpolar fluid. The concentration of diatomic molecules is assumed to be low enough so that a diatomic molecule (absorber) interacts with the solvent molecules (perturbers) independently of other diatomics. Under this condition the linear response theory gives the FIR absorption coefficient per unit path length, $\alpha(\omega)$, of a sample at temperature $T$, as [11]

$$
\alpha(\omega)=\frac{4 \pi \rho_{A} \omega}{3 \hbar c} f(n)\left(1-e^{-\beta \hbar \omega}\right) \operatorname{Re}\left\{\int_{0}^{\infty} d t e^{i \omega t}\langle\overrightarrow{\mathrm{M}}(t) \cdot \overrightarrow{\mathrm{M}}(0)\rangle\right\}
$$


where $\rho_{A}$ is the number density of absorbers, $f(n)=(1 / n)\left(n^{2}+2\right)^{2} / 9$ is the Polo-Wilson correcting factor for internal field effects ( $n$ is the optical refractive index) [6], $\beta=1 /\left(k_{B} T\right), \overrightarrow{\mathrm{M}}(t)$ is the dipole moment operator in the Heisenberg picture of the system associated to the presence of each diatomic molecule in the solution, and the angular brackets denote a canonical equilibrium average. In Eq. (1), the refractive index, $n$, can be obtained from the Lorenz-Lorenz equation [12] $\left(n^{2}-1\right) /\left(n^{2}+2\right)=(4 \pi / 3) \rho_{B} \alpha_{B}$, where $\rho_{B}$ is the number density of the solvent molecules and $\alpha_{B}$ its spherical polarizability.

The dipole moment is the sum of the permanent dipole moment of the diatomic molecule and the induced dipole moment due to the diatomicsolvent interaction. Assuming that the induced dipole moment arises from a sum of binary diatom-atom interactions, one can write,

$$
\overrightarrow{\mathrm{M}}(t)=\vec{\mu}_{\mathrm{p}}[\vec{r}(t)]+\sum_{k \in \text { solvent }} \vec{\mu}\left[\vec{r}(t), \vec{R}_{k}(t)\right]
$$

where $\vec{\mu}_{\mathrm{p}}(\vec{r})$ is the permanent dipole moment of the diatomic, $\vec{\mu}\left(\vec{r}, \vec{R}_{k}\right)$ represents the induced dipole moment in a diatom-atom collision, $\vec{r}=(r, \Omega)$ is the vector describing the internuclear separation and the orientation of the diatomic molecule, and $\overrightarrow{R_{k}}=\left(R_{k}, \Omega_{k}\right)$ is the vector joining the centers of mass of the $k$-th solvent molecule and the diatomic one. This assumption implies that eventual irreducible triplet or higher order dipole moments are neglected. In the FIR, the vibrational degrees of freedom are not optically active and we can suppose that the diatomic molecule remains on its ground vibrational state. Then, the dependence on $r$ in Eq. (2) disappears. Besides, the induced dipole moment can be expanded in terms of spherical harmonics in the following way [13]:

$$
\begin{aligned}
& \mu_{M}\left(\Omega, \vec{R}_{k}\right)= \\
& \frac{4 \pi}{\sqrt{3}} \sum_{\lambda, L} B_{\lambda, L}\left(R_{k}\right) \sum_{m_{\lambda}, m_{L}} C\left(\lambda, L, 1 ; m_{\lambda}, m_{L}, M\right) Y_{L}^{m_{L}}\left(\Omega_{k}\right) Y_{\lambda}^{m_{\lambda}}(\Omega)
\end{aligned}
$$

where the subscript $M=-1,0,1$ denotes spherical tensor components of the dipole moment, $C\left(\lambda, L, 1 ; m_{\lambda}, m_{L}, M\right)$ are Clebsch-Gordan coefficients, $Y$ denotes spherical harmonics, and $B_{\lambda, L}\left(R_{k}\right)$ are radial expansion coefficients specifying various dipole induction mechanisms.

The analytical calculation of the absorption coefficient from Eqs. (1)-(3) is unattainable without the consideration of some simplifying hypotheses. We would like to mention the three main assumptions considered in our works (besides the pairwise additivity of the intermolecular interactions):

1) The induction mechanisms responsible for the absorption in the system under study are essentially electrostatic in nature. With this assumption only radial coefficients, $B_{\lambda, \lambda+1}(R)$, will occur in the expansion (3) of 
the induced dipole moment, and one has [14]-[16]

$$
B_{\lambda, L}\left(R_{k}\right)=\sqrt{\lambda+1} \frac{\alpha_{B} Q_{\lambda}}{R_{k}^{\lambda+2}} \delta_{\lambda+1, L} \quad(\text { for } \lambda \geq 1)
$$

where $Q_{\lambda}$ is the $\lambda$-order multipole moment of the diatomic molecule (i.e., $Q_{1} \equiv \mu_{\mathrm{p}}, Q_{2} \equiv \Theta, Q_{3} \equiv \Omega$, and $Q_{4} \equiv \Phi$ are, respectively, the permanent dipole, quadrupole, octupole and hexadecapole moments). Note that, in general, $B_{\lambda, \lambda+1}(R)$ has both an overlap (short range) and an electric multipole (long range) contribution. The asymptotic approximation (4) seems to be adequate for low-density gases and highly polarizable solvents, for which the induction mechanisms are fundamentally long range, and can be used as a reasonable starting point for the evaluation of the multipole induced effects in high-density gases and liquids.

2) We consider that the dynamics of the total system is governed by the Hamiltonian $H=H_{R}+H_{B}+H^{\prime}$, where $H_{R}$ is the free Hamiltonian for the rotational motion of the diatomic molecule (system $R$ ), $H_{B}$ is the Hamiltonian for the translational motions of the solute and solvent molecules (system $B$ ), and $H^{\prime}$ is the Hamiltonian describing the interaction $R-B$. Then, we assume that the translational degrees of freedom are not affected by the rotational motion of the diatomic molecule, i.e., by the anisotropic part of the interaction Hamiltonian $H^{\prime}$, but the opposite does not hold and the translational part behaves as a thermal bath for the rotational relaxation. We shall refer to this assumption as the translational decoupling approximation. It allows to perform separate averages in Eq. (1), but it breaks down if the density fluctuations in the fluid give rise to large, rapid fluctuations of the electric field at the site of the diatomic molecule [17].

3) As carefully studied by Borysow et al. [18], spectral moments have in general two kinds of contributions: the pure terms coming from each spherical component of the induced dipole moment and the mixing terms coming from the superposition of induced dipole spherical components of different symmetry. Strictly, last terms vanish only when interactions are considered isotropic. We have restricted our study to the spectral contributions arising from pure electric induced terms.

The above assumptions allows us to express the FIR absorption coefficient (1) as the sum of a permanent (P) and an electrostatic induced (I) contribution,

$$
\alpha(\omega)=\alpha^{(P)}(\omega)+\alpha^{(I)}(\omega)
$$

with

$$
\alpha^{(P)}(\omega)=\frac{4 \pi \rho_{A} \omega}{3 \hbar c} f(n)\left(1-e^{-\beta \hbar \omega}\right) \mu_{p}^{2} \widehat{C}_{r o t}^{(1)}(\omega),
$$




$$
\alpha^{(I)}(\omega)=\sum_{\lambda=1}^{\infty} \alpha^{\left(Q_{\lambda}\right)}(\omega)
$$

where $\alpha^{\left(Q_{\lambda}\right)}(\omega)$ is given by the convolution of a $\lambda$-translational spectrum and a $\lambda$-rotational spectrum,

$\alpha^{\left(Q_{\lambda}\right)}(\omega)=\frac{4 \rho_{A} \omega}{3 \hbar c} f(n)\left(1-e^{-\beta \hbar \omega}\right) \int_{-\infty}^{\infty} \widehat{C}_{t r}^{(\lambda, \lambda+1)}(\omega) \widehat{C}_{\text {rot }}^{(\lambda)}\left(\omega-\omega^{\prime}\right) \mathrm{d} \omega^{\prime}$,

where

$$
\begin{aligned}
\widehat{C}_{t r}^{(\lambda, \lambda+1)}(\omega) & \equiv \operatorname{Re}\left\{\int_{0}^{\infty} d t e^{i \omega t} C_{t r}^{(\lambda, \lambda+1)}(t)\right\}, \\
\widehat{C}_{\text {rot }}^{(\lambda)}(\omega) & \equiv \operatorname{Re}\left\{\int_{0}^{\infty} d t e^{i \omega t} C_{\text {rot }}^{(\lambda)}(t)\right\}
\end{aligned}
$$

with

$$
\begin{gathered}
C_{t r}^{(\lambda, \lambda+1)}(t)=\left\langle\sum_{k, k^{\prime}} B_{\lambda, \lambda+1}\left(R_{k}(t)\right) B_{\lambda, \lambda+1}\left(R_{k^{\prime}}(0)\right) P_{\lambda+1}\left(\cos \theta_{k, k^{\prime}}(t)\right)\right\rangle, \\
C_{\text {rot }}^{(\lambda)}(t)=\frac{4 \pi}{2 \lambda+1} \sum_{m_{\lambda}}(-1)^{m_{\lambda}}\left\langle\left\langle Y_{\lambda}^{m_{\lambda}}(\Omega(t)) Y_{\lambda}^{-m_{\lambda}}(\Omega(0))\right\rangle\right\rangle
\end{gathered}
$$

where $P_{L}$ are the Legendre Polynomials, $\theta_{k, k^{\prime}}$ is the angle between $\overrightarrow{R_{k}}$ and $\overrightarrow{R_{k^{\prime}}},\langle\cdots\rangle$ denote a canonical equilibrium average over the translational variables, and $\langle\langle\cdots\rangle\rangle$ denote a canonical equilibrium average over both translational and rotational degrees of freedom.

In the case of homonuclear diatomic molecules there is no permanent contribution and only induced contributions with $\lambda$ even must be taken into account in Eq. (7).

\subsection{TRANSLATIONAL SPECTRAL CONTRIBUTION}

For low- and moderate-density gas mixtures the translational spectral contribution $\widehat{C}_{t r}^{(\lambda, \lambda+1)}(\omega)$ can be successfully calculated using the ZwanzigMori (or memory function) approach [19]-[21] based on a continued-fraction representation for the Laplace transform of the translational time-correlation function (11), truncated at the first or second stage, and assuming a Gaussian form for the corresponding memory function. This enables one to obtain the first three even spectral moments of $\widehat{C}_{t r}^{(\lambda, \lambda+1)}(\omega)$. In particular, 
the zeroth order moment (the integrated intensity) is given by

$$
\begin{aligned}
C_{\lambda, \lambda+1} & \equiv C_{t r}^{(\lambda, \lambda+1)}(0)=\rho_{B} \int g_{A B}\left(R_{k}\right)\left[B_{\lambda, \lambda+1}\left(R_{k}\right)\right]^{2} d^{3} \vec{R}_{k} \\
& +\rho_{B}^{2} \iint g_{A B B}\left(R_{k}, R_{k^{\prime}}, R_{k, k^{\prime}}\right) \times \\
& \times B_{\lambda, \lambda+1}\left(R_{k}\right) B_{\lambda, \lambda+1}\left(R_{k^{\prime}}\right) P_{\lambda+1}\left(\cos \theta_{k, k^{\prime}}\right) d^{3} \vec{R}_{k} d^{3} \vec{R}_{k^{\prime}}
\end{aligned}
$$

where $g_{A B}\left(R_{k}\right)$ and $g_{A B B}\left(R_{k}, R_{k^{\prime}}, R_{k, k^{\prime}}\right)$ are, respectively, the two- and three-body distribution functions, and $R_{k, k^{\prime}}=\left|\vec{R}_{k}-\vec{R}_{k^{\prime}}\right|$.

For low-gas density systems, the pair distribution functions $g_{A B}\left(R_{k}\right)$ and $g_{B B}\left(R_{k, k^{\prime}}\right)$ can be evaluated from the low density limit expressions:

$g_{A B}\left(R_{k}\right)=\exp \left[-\frac{V_{0}^{(A B)}\left(R_{k}\right)}{k T}\right] ; g_{B B}\left(R_{k, k^{\prime}}\right)=\exp \left[-\frac{V_{0}^{(B B)}\left(R_{k, k^{\prime}}\right)}{k T}\right]$

where $V_{0}^{(A B)}\left(R_{k}\right)$ and $V_{0}^{(B B)}\left(R_{k, k^{\prime}}\right)$ are the respective solute-solvent and solvent-solvent isotropic intermolecular potentials, while the three-body distribution function can be evaluated in the Kirkwood superposition approximation [22]:

$$
g_{A B B}\left(R_{k}, R_{k^{\prime}}, R_{k, k^{\prime}}\right)=g_{A B}\left(R_{k}\right) g_{A B}\left(R_{k^{\prime}}\right) g_{B B}\left(R_{k, k^{\prime}}\right),
$$

with $g_{A B}\left(R_{k}\right)$ and $g_{B B}\left(R_{k, k^{\prime}}\right)$ given by Eqs. (14).

A similar formalism can also be applied to calculate the translational spectral contributions for high-density gas and liquid phases, but one then needs the knowledge of appropriate two- and three-body distribution functions. Guillot et al. [21, 23] have used this method for the study of the FIR spectrum of dense $\mathrm{N}_{2}$ and $\mathrm{N}_{2}$-rare gas compressed mixtures. These authors performed the calculation of the spectral moments only for the quadrupoleinduced dipole contribution. In their work the two-body distribution function was evaluated from the analytical density dependent approximation provided by Chesnoy [24], whereas the three-body contributions were evaluated making use of a lattice-gas model. Within this model the three-body distribution functions were implemented by assuming that a multiple occupation of a given site of the lattice is forbidden, all the remaining locations being equally probable. Values of the spectral moments obtained by these authors allow for satisfactorily reproducing experimental FIR spectra for systems with density less than about 650 amagat. However, for systems at higher densities the predicted moments disagree with the experimental ones.

Furthermore, we also intend to evaluate the effect of other (higher than quadrupole) multipole-induced dipole contributions. Since each of these 
contributions has a characteristic range of interaction, a suitable evaluation of the two- and three-body distribution functions is needed in each case. Concretely, if the lattice-gas model is used to evaluate the three-body contributions, the weight of each configuration must be different in order to account for the particular range of the considered multipole-induced contribution. The latter complicates the abovementioned method for the calculation of the moments for the different multipole-induced dipole contributions. Therefore, in our work, the translational functions $\widehat{C}_{t r}^{(\lambda, \lambda+1)}(\omega)$ for the high-density gas and liquid solutions have been obtained from molecular dynamics (MD) calculations.

\subsection{ROTATIONAL SPECTRAL CONTRIBUTION}

The $\lambda$-rotational contribution $\widehat{C}_{\text {rot }}^{(\lambda)}(\omega)$ was calculated in the framework of a quantum theory describing the rotational dynamics of the diatomic molecule. This theory is based upon the following assumptions:

(i) The unperturbed rotational states of the diatomic (system $R$ ) are taken as the eigenstates of a free quantum rigid rotor, $\left\{|i>\equiv| j_{i}, m_{i}>\right\}$, with eigenvalues $E_{j_{i}}=B h c j_{i}\left(j_{i}+1\right)$, where $B$ is the rotational constant of the diatomic molecule on its ground vibrational state, and other symbols have their usual meaning. A non-rigid rotor including centrifugal distortion of the solute molecules has been also considered [25]. Deviations from the rigid rotor model become noticeable only for the transitions from the eigenstates with large enough values of $j$.

(ii) All translational degrees of freedom, both of absorber and perturber species, behave as a classical thermal bath (system $B$ ) at the temperature $T$ of the solution.

(iii) The anisotropic part of the absorber-perturber intermolecular potential $V$ is given by a truncated series in Legendre polynomials, in the form

$$
V=\sum_{k \in \text { solvent }} \sum_{J=1}^{J_{\max }} V_{J}\left(R_{k}\right) P_{J}\left(\cos \Omega_{k}\right),
$$

where $\Omega_{k}$ is the angle between $\vec{R}_{k}$ and the diatomic internuclear axis. Then, the interaction hamiltonian $H^{\prime}$ is given by $H^{\prime}=V-\langle V\rangle$.

(iv) The time evolution of the radial part $V_{J}\left(R_{k}\right)$ of the intermolecular potential (16) is described by means of a classical stochastic process with a time autocorrelation function (TCF) given by

$$
\Phi_{J}(t)=\left\langle V_{J}\left(R_{k}(t)\right) V_{J}\left(R_{k}(0)\right)\right\rangle-\left\langle V_{J}\left(R_{k}(t)\right)\right\rangle^{2}=\kappa_{J}^{2} \exp \left[-|t| / t_{J}\right],
$$

where $\kappa_{J}$ and $t_{J}$ are characteristic parameters of the system measuring the mean strength of the anisotropic $J$ contribution to the potential (16) and its correlation time, respectively. 
Using these assumptions, it has been shown elsewhere that the $\lambda$-rotational contribution $\widehat{C}_{\text {rot }}^{(\lambda)}(\omega)$ may be written as a sum of two contributions [26]

$$
\widehat{C}_{\text {rot }}^{(\lambda)}(\omega)=\widehat{C}_{0, \text { rot }}^{(\lambda)}(\omega)+\widehat{C}_{1, \text { rot }}^{(\lambda)}(\omega)
$$

The first one, called secular profile, has the form

$$
\widehat{C}_{0, \text { rot }}^{(\lambda)}(\omega)=\sum_{j_{i}} \sigma_{j_{i}}^{o} \sum_{j_{f}}\left(2 j_{f}+1\right) A_{j_{f}, j_{i}}(\lambda) \operatorname{Re}\left\{\Lambda_{j_{f}, j_{i}}^{(\lambda)}(\omega)\right\}
$$

where $\sigma_{j_{i}}^{o}$ is the usual rotational population factor of state $\left|j_{i}\right\rangle$, the coefficients $A_{j_{f}, j_{i}}(\lambda)$ are given by

$$
A_{j, j^{\prime}}(\lambda)=\left(2 j^{\prime}+1\right)\left(\begin{array}{ccc}
j & \lambda & j^{\prime} \\
0 & 0 & 0
\end{array}\right)^{2}
$$

and

$$
\Lambda_{j_{f}, j_{i}}^{(\lambda)}(\omega)=\frac{i}{\omega-\omega_{j_{f}, j_{i}}+i \bar{W}_{j_{f}, j_{i}}^{(\lambda)}(\omega)}
$$

with $\bar{W}_{j_{f}, j_{i}}^{(\lambda)}(\omega)=\sum_{J=1}^{J_{\max }} \bar{W}_{j_{f}, j_{i}}^{(\lambda, J)}(\omega)$. Explicit expressions for the functions $\bar{W}_{j_{f}, j_{i}}^{(\lambda, J)}(\omega)$ are given in Ref. [26] in terms of the Fourier-Laplace transform of the TCF (17).

The second contribution $\widehat{C}_{1, r o t}^{(\lambda)}(\omega)$ in (18), called interference profile, has the form:

$$
\widehat{C}_{1, \text { rot }}^{(\lambda)}(\omega)=\sum_{J=1}^{J_{\max }} \widehat{C}_{1, \text { rot }}^{(\lambda, J)}(\omega)
$$

Explicit expressions for the anisotropic contributions $\widehat{C}_{1, \text { rot }}^{(\lambda, J)}(\omega)$ are reported in Ref. [26] in terms of the rotational lines (21).

The secular approximation becomes invalid [27, 28] when the spectrum is significantly affected by the overlap among the basic secular resonances $\left(\Lambda_{j_{f}, j_{i}}^{(\lambda)}(\omega)\right.$ and $\left.\Lambda_{j_{f^{\prime}}, j_{i^{\prime}}}^{(\lambda)}(\omega)\right)$ causing an intensity redistribution inside the absorption band. This line-mixing (nonadditivity) effect is taken into account by means of the interference contribution, $\widehat{C}_{1, r o t}^{(\lambda)}(\omega)$ [Eq.(22)]. The number of basic resonances involved in the interference depends on the order of Legendre polynomials in the interaction Hamiltonian.

Besides interference contribution, the spectral theory incorporates nonmarkovian (or memory) effects that mathematically stem from the $\omega$-dependence of the basic resonances. When the markovian approach is valid, each secular resonance $\Lambda_{j_{f} j_{i}}^{(\lambda)}(\omega)$ becomes a Lorentzian line with halfwidth 
given by $\operatorname{Re}\left[\bar{W}_{j_{f} j_{i}}^{(\lambda)}\left(\omega_{j_{f} j_{i}}\right)\right]$. From the point of view of the involved solutesolvent autocorrelation functions, this means that the autocorrelation time $t_{J}$ of $\Phi_{J}(t)$ is much smaller that the inverse of the mean-square interaction strength, $\kappa_{J} t_{J} / h<<1[27,28]$.

\section{Far-infrared spectra of $\mathrm{CO}$ in Ar}

Comparison between theoretical and experimental FIR spectra of HCl-rare gas solutions at different thermodynamic states show that the permanent dipole contribution is sufficient to explain the whole spectrum of this system both at low and high densities [27]. Roco et al. [3] have also applied the outlined spectral theory to investigate the FIR absorption spectra of CO in $\mathrm{Ar}$ gas at various densities. Comparison with the experimental spectra measured by Buontempo et al. [4] showed that, as in the case of HClrare gas mixtures, the permanent dipolar contribution is overwhelming at low-gas densities. However, at difference of HCl-rare gas cases, as the Ar density increases the permanent dipolar contribution does not account for the observed spectrum, specially for the existence of a long tail extending towards the high frequency range. This fact clearly shows the existence of significant induced dipole contributions to the FIR absorption spectra of CO-Ar solutions as density increases.

The spectral theory has been applied to calculate the FIR spectra of CO dissolved in $\mathrm{Ar}$ at three different thermodynamic states: 1) a low-density gas solution at $T=129 \mathrm{~K}$ and $13 \times 10^{20} \mathrm{~cm}^{-3}$ (48.3 amagat); 2) a highdensity gas at $T=152 \mathrm{~K}$, and $\rho_{\mathrm{Ar}}=118 \times 10^{20} \mathrm{~cm}^{-3}$ (438.7 amagat); and 3 ) a liquid solution at $T=90 \mathrm{~K}$ and $\rho_{\mathrm{Ar}}=200 \times 10^{20} \mathrm{~cm}^{-3}$ (743.6 amagat). Evaluation of the FIR absorption spectra of CO-Ar at the considered temperatures and densities requires the knowledge of the multipole moments of $\mathrm{CO}$ on its ground vibrational state. These parameters have been subject of many experimental and theoretical investigations, and the results cover a moderately wide range of values. In this context, a delicate point is the fact that, although the absolute value of the electric dipole moment is independent of the location of the coordinate origin, all other multipole moments are not reference-frame invariant. Consequently, a shift of the coordinate origin from, say, the center of mass to the center of charge changes the values of all $\left|Q_{\lambda}\right|$ with $\lambda \geq 2$. The values available in the literature for the molecular multipole moments of $\mathrm{CO}$ lie in the following ranges

$$
\begin{array}{ll}
\left|\mu_{p}\right|=[0.1096,0.112] \mathrm{D} & |\Theta|=[0.92,2.81] \mathrm{D} \AA \\
|\Omega|=[2.34,3.56] \mathrm{D} \AA^{2} & |\Phi|=[2.92,4.74] \mathrm{D} \AA^{3} .
\end{array}
$$

In the present work, we consider these ranges for a first estimation of the translational strength parameters $C_{\lambda, \lambda+1}$. However, for comparison pur- 


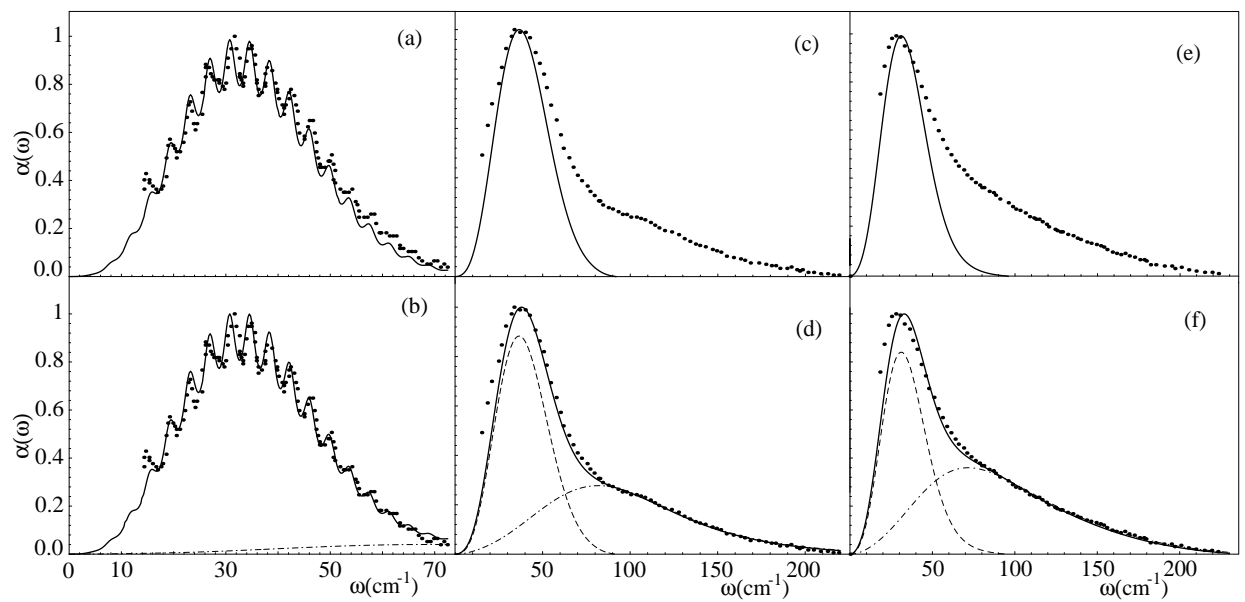

Figure 1. FIR spectra of CO in Ar at: $T=129 \mathrm{~K}$ and $n_{\mathrm{A} r}=13 \times 10^{20} \mathrm{~cm}^{-3}[(\mathrm{a})$ and (b)], $T=152 \mathrm{~K}$, and $n_{\mathrm{A} r}=118 \times 10^{20} \mathrm{~cm}^{-3}[(\mathrm{c})$ and $(\mathrm{d})]$, and $T=90 \mathrm{~K}$, and $n_{\mathrm{A} r}=200 \times 10^{20}$ $\mathrm{cm}^{-3}[(\mathrm{e})$ and (f)]. (a), (c), and (e): Theoretical profiles obtained considering only the permanent dipole contribution (solid line). (b), (d), and (f): Theoretical profiles (solid line) obtained as a sum of the permanent (dashed line) and the induced contributions (dash-dotted line). Experimental profiles (dotted lines).

poses, we take $\left|\mu_{p}\right|=0.1097 \mathrm{D}$ while we consider the other multipole moments as adjustable parameters. An estimation of $|\Theta|,|\Omega|$ and $|\Phi|$ can be then made by resolving the FIR absorption spectra of $\mathrm{CO}$ in Ar into a permanent and a multipole-induced dipole absorption band and by fitting to the experimental profiles.

The theoretical spectra are obtained from the expressions reported in the previous Section with $B_{\mathrm{CO}}=1.92 \mathrm{~cm}^{-1}[4], \alpha_{\mathrm{Ar}}=1.62 \AA^{3}[32]$, and $J_{\max }=2$. Then, taking into account Eq. (17), four parameters $\left(\kappa_{1}, \kappa_{2}, t_{1}\right.$, $t_{2}$ ) are required in order to evaluate the theoretical FIR absorption coefficient. These parameters are considered as a set of adjustable parameters whose values are chosen by comparison with experimental spectra.

Experimental FIR $\left(15-75 \mathrm{~cm}^{-1}\right)$ spectrum of $\mathrm{CO}$ in Ar gas at low enough densities present a well resolved rotational structure. Calculated and experimental absorption coefficients are represented in Fig. 1. Since the experimental solute number density $\rho_{\mathrm{CO}}$ is unknown, each spectrum is normalized to the respective maximum value. The theoretical absorption coefficient obtained just considering the permanent contribution is represented in Fig. 1.a. This permanent contribution, $\alpha^{(P)}(\omega)$, has been calculated from Eq. (6). From Fig. 1.a it is clear that the low-density gas FIR spectrum of this system is essentially due to permanent dipole absorption. 
However, on the high-frequency region it is observed that the theoretical permanent FIR spectrum underestimates experimental absorption. It is reasonable to assume that this slight discrepancy can be evaluated in terms of the induced absorption band.

\begin{tabular}{cccc}
\hline & $(1)$ & $(2)$ & $(3)$ \\
\hline$C_{1,2} / \mu_{p}^{2}$ & $1.21 \times 10^{-3}$ & $3.73 \times 10^{-3}$ & $2.24 \times 10^{-3}$ \\
$C_{2,3} / \Theta^{2}\left(\AA^{-2}\right)$ & $1.00 \times 10^{-4}$ & $4.52 \times 10^{-4}$ & $4.07 \times 10^{-4}$ \\
$C_{3,4} / \Omega^{2}\left(\AA^{-4}\right)$ & $9.30 \times 10^{-6}$ & $5.88 \times 10^{-5}$ & $7.50 \times 10^{-5}$ \\
$C_{4,5} / \Phi^{2}\left(\AA^{-6}\right)$ & $8.50 \times 10^{-7}$ & $6.87 \times 10^{-6}$ & $1.32 \times 10^{-5}$ \\
\hline
\end{tabular}

TABLE 1 . Values of the parameters $C_{\lambda, \lambda+1} / Q_{\lambda}^{2}$ for CO-Ar: (1) $T=129 \mathrm{~K}$ and $n_{A r}=13 \times 10^{20} \mathrm{~cm}^{-3}$ (calculated from the Zwanzig-Mori approach); (2) $T=152 K$ and $n_{A r}=118 \times 10^{20}$ $\mathrm{cm}^{-3}$ (MD simulations); (3) $T=90 \mathrm{~K}$ and $n_{A r}=200 \times 10^{20}$ $\mathrm{cm}^{-3}$ (MD simulations).

In order to evaluate the significant induced contributions to the absorption profile we have calculated the translational strength parameters $C_{\lambda, \lambda+1} / Q_{\lambda}^{2}$ from Eq. (13) by using the long-range induction functions (4) and the two- and three-body radial distribution funtions (14) and (15) with isotropic intermolecular potentials $V_{0}^{(A B)}\left(R_{k}\right)$ and $V_{0}^{(B B)}\left(R_{k, k^{\prime}}\right)$ taken as $6-12$ Lennard-Jones surfaces with parameters [6]: $\sigma_{\mathrm{CO}-\mathrm{Ar}}=3.483 \AA$, $\epsilon_{\mathrm{CO}-\mathrm{Ar}}=117.06 \mathrm{~K}$, and $\sigma_{\mathrm{Ar}-\mathrm{Ar}}=3.405 \AA, \epsilon_{\mathrm{Ar}-\mathrm{Ar}}=119.8 \mathrm{~K}$. The calculated values are given by the first column of Table 1 . We note the following points: a) $C_{1,2} / \mu_{p}^{2} \ll 1$, which indicates that the dipole-induced dipole contribution can be neglected; b) since the permanent contribution presents a maximum absorption at the rotational transition line $j=7 \rightarrow 8$, the maximum absorption of the quadrupole-, octupole- and hexadecapoleinduced dipole contributions respectively occurs at $\omega \approx 65 \mathrm{~cm}^{-1}\left[\omega_{j+2, j}=\right.$ $2 B(2 j+3)$ with $j=7], \omega \approx 104 \mathrm{~cm}^{-1}\left[\omega_{j+3, j}=6 B(j+2)\right.$ with $\left.j=7\right]$, and $\omega \approx 146 \mathrm{~cm}^{-1}\left[\omega_{j+4, j}=4 B(2 j+5)\right.$ with $\left.j=7\right]$, then, one can assume that in the observed frequency range $\left(15-75 \mathrm{~cm}^{-1}\right)$, the octupole $(\lambda=3)$ and hexadecapole $(\lambda=4)$ contributions can be also neglected. Thus, the FIR spectrum of CO in Ar gas at low density has been obtained from the sum of permanent and quadrupole-induced dipole contributions,

$$
\alpha(\omega)=\alpha^{(P)}(\omega)+\alpha^{(\Theta)}(\omega) .
$$

where $\alpha^{(P)}(\omega)$ has been evaluated from Eq. (6) and $\alpha^{(\Theta)}(\omega)$ from Eq. (8) for $\lambda=2$. The translational spectral function $\widehat{C}_{t r}^{(2,3)}(\omega)$ was calculated 
using the Zwanzig-Mori approach while the rotational spectral functions $\widehat{C}_{\text {rot }}^{(1)}(\omega)$ and $\widehat{C}_{\text {rot }}^{(2)}(\omega)$ were calculated from Eq. (18). The best agreement with the experimental lineshapes was obtained for $\kappa_{1}=4.5 \times 10^{-22} \mathrm{~J}$, $\kappa_{2}=8.0 \times 10^{-22} \mathrm{~J}, t_{1}=t_{2}=1.1 \times 10^{-14} \mathrm{~s}$, and $C_{2,3}=3.80 \times 10^{-4} \mathrm{D}^{2}$. Taking into account the value of $C_{2,3} / \Theta^{2}$ reported in the first column of Table 1 , this fit leads to

$$
|\Theta|=1.95 \mathrm{D} \AA
$$

which favorably compares with the result 1.94(4) D $\AA$ measured by Meerts et al. [29] by using a molecular beam electric resonance method. In Fig. 1.b we have plotted the theoretical spectrum obtained from Eq. (24), with $\Theta$ given by (25). As can be observed in this figure, the above discrepancies between permanent and experimental profiles disappear when the quadrupole-induced contribution is considered.

The FIR $\left(15-220 \mathrm{~cm}^{-1}\right)$ spectra of $\mathrm{CO}$ in $\mathrm{Ar}$ gas at $T=152 \mathrm{~K}$ and $\rho_{\mathrm{Ar}}=118 \times 10^{20} \mathrm{~cm}^{-3}$ and in Ar liquid at $T=90$ and $\rho_{\mathrm{Ar}}=200 \times 10^{20}$ $\mathrm{cm}^{-3}$ have been also measured by Buontempo et al. [4] and they are devoid of any rotational structure. The corresponding calculated and experimental absorption coefficients are also represented in Fig. 1. As before, the spectra are normalized to the respective maximum value of the total spectrum considered in each case. The theoretical absorption coefficients obtained by considering only the permanent contribution, $\alpha^{(P)}(\omega)$, are represented in Figs. 1.c and 1.e. These figures clearly show that, in both cases, permanent dipole absorption can not account for the whole FIR profile. This is particularly valid for the long tail appearing at intermediate and high frequencies, and becomes more evident as solvent density increases.

We have calculated the FIR spectra at these conditions as the sum of the permanent contribution and an electrostatic induced contribution including its dipole, quadrupole, octupole and hexadecapole components,

$$
\begin{aligned}
\alpha(\omega) & =\alpha^{(P)}(\omega)+\alpha^{(I)}(\omega) \\
\alpha^{(I)}(\omega) & =\alpha^{(\mu)}(\omega)+\alpha^{(\Theta)}(\omega)+\alpha^{(\Omega)}(\omega)+\alpha^{(\Phi)}(\omega)
\end{aligned}
$$

where the induced contributions, $\alpha^{(\mu)}(\omega), \alpha^{(\Theta)}(\omega), \alpha^{(\Omega)}(\omega)$ and $\alpha^{(\Phi)}(\omega)$ have been obtained from Eq. (8) with $\lambda=1,2,3$, and 4 .

Because of the difficulties in applying the Zwanzig-Mori approach to high density systems, the translational functions $\widehat{C}_{t r}^{(\lambda, \lambda+1)}(\omega)$ for the highdensity gas and liquid cases have been obtained from MD calculations. In all computer simulations we consider a sample of 250 solvent atoms (Ar) and one diatomic molecule (CO) in a cubic box with the usual boundary conditions. The $\mathrm{CO}$ molecule has been considered as isotropic and the interactions (CO-Ar and Ar-Ar) were considered to be of the Lennard-Jones type with parameters previously reported. The equations of motion were 
integrated by means of a leap-frog Verlet algorithm with coupling to a thermal bath to keep the system temperature at the desired values. The time step was $\Delta t=0.5 \times 10^{-14} \mathrm{~s}$. After a long equilibration period, the dynamics of the particles was followed during $8 \times 10^{5}$ time steps in all cases, and the averages of the time correlation functions relevant for the problem were performed. The values of the four leading $(\lambda=1,2,3$, and 4$)$ strength parameters $C_{\lambda, \lambda+1} / Q_{\lambda}^{2}$ obtained from MD calculations are listed Table 1.

The rotational spectral functions $\widehat{C}_{\text {rot }}^{(\mu)}(\omega), \widehat{C}_{\text {rot }}^{(\Theta)}(\omega), \widehat{C}_{\text {rot }}^{(\Omega)}(\omega)$, and $\widehat{C}_{\text {rot }}^{(\Phi)}(\omega)$ were calculated from Eq. (18) and with values of the statistical parameters $\kappa_{1}=10.1 \times 10^{-22} \mathrm{~J}, \kappa_{2}=16.2 \times 10^{-22} \mathrm{~J}$, and $t_{1}=t_{2}=$ $1.1 \times 10^{-14} \mathrm{~s}$ for the high-density gas case, and $\kappa_{1}=13.2 \times 10^{-22} \mathrm{~J}, \kappa_{2}=$ $20.9 \times 10^{-22} \mathrm{~J}$, and $t_{1}=t_{2}=1.1 \times 10^{-14} \mathrm{~s}$ for the liquid.

In Fig. 1.d we have plotted the overall absorption coefficient for the high-density gas case with $C_{1,2}=4.49 \times 10^{-5} \mathrm{D}^{2}, C_{2,3}=2.03 \times 10^{-3} \mathrm{D}^{2}$, $C_{3,4}=5.15 \times 10^{-4} \mathrm{D}^{2}$ and $C_{4,5}=6.47 \times 10^{-5} \mathrm{D}^{2}$, which give the best agreement with the experimental profile. Taking into account the values of $C_{2,3} / \Theta^{2}, C_{3,4} / \Omega^{2}$ and $C_{4,5} / \Phi^{2}$ given in the second column of Table 1 , this fit leads to:

$$
|\Theta|=2.12 \mathrm{D} \AA, \quad|\Omega|=2.96 \mathrm{D}^{2}, \quad|\Phi|=3.07 \mathrm{D} \AA^{3} .
$$

The value of $|\Theta|$ is within the experimental range (23), but it is a $7.7 \%$ larger than the value (25) obtained from the low-density gas case. The value of $|\Omega|$ is in excellent agreement with the theoretical values reported by Diercksen and Sadlej [30] and by Bounds and Wilson [31], while the value of $|\Phi|$ is in the low region of the interval (23).

Fig. 1.f contains the theoretical shape of $\alpha(\omega)$ for the liquid case with $C_{1,2}=2.61 \times 10^{-5} \mathrm{D}^{2}, C_{2,3}=2.08 \times 10^{-3} \mathrm{D}^{2}, C_{3,4}=7.54 \times 10^{-4} \mathrm{D}^{2}$ and $C_{4,5}=5.75 \times 10^{-5} \mathrm{D}^{2}$, which give the best agreement with the experimental profile. Taking into account the values of $C_{2,3} / \Theta^{2}, C_{3,4} / \Omega^{2}$ and $C_{4,5} / \Phi^{2}$ given in the third column of Table 1 , this fit leads to:

$$
|\Theta|=2.26 \mathrm{D} \AA, \quad|\Omega|=3.17 \mathrm{D} \AA^{2}, \quad|\Phi|=6.60 \mathrm{D} \AA^{3} .
$$

The value of $|\Theta|$ is still into the range (23), but it is a $16 \%$ greater than the value (26) obtained from the fit of the low-density gas spectrum. The value of $|\Omega|$ is also into the range (23), and it is a $7 \%$ greater than the value (27) obtained from the fit of the high-density gas spectrum. Conversely, the value of $|\Phi|$ is now higher than the reported by Bounds and Wilson [31] and it is 2.15 times the value (27) obtained from the fit of the high-density gas spectrum.

As we have commented before, the consideration of only long-ranged induction mechanisms could be a reasonable assumption for low gas densities. However, when the density increases, other contributions could be 
significantly involved in the FIR absorption spectrum. For example, the influence of short ranged (overlap) induction mechanisms for the liquid nitrogen has been pointed out by Guillot et al. $[6,23]$. Their consideration in our work would require to carry out first principles calculations in order to provide expressions for the involved functions $B_{\lambda, \lambda \pm 1}$. Furthermore, since the hexadecapole-induced dipole contribution is specially significant in the high-frequency region, the calculated increasing of $\Phi$ at the liquid density can be also attributed to higher order multipole mechanisms or to spectral manifestations of triplet induced dipoles in this particular frequency region. Therefore, in our spectral theory, the multipole moments $\left|Q_{\lambda}\right|$ must be considered as effective parameters, providing all the induced mechanisms which have not been considered.

\section{Far-infrared spectra of $\mathrm{N}_{2}$ in $\mathrm{Xe}$}

The theory outlined in Sec. II was also applied to describe the FIR-spectrum $\left(50-250 \mathrm{~cm}^{-1}\right)$ of a $\mathrm{N}_{2}-\mathrm{Xe}$ gaseous mixture at $T=295 \mathrm{~K}, \rho_{\mathrm{N}_{2}}=3$ amagat $\left(0.81 \times 10^{20} \mathrm{~cm}^{-3}\right)$ and $\rho_{\mathrm{Xe}}=350$ amagat $\left(94.15 \times 10^{20} \mathrm{~cm}^{-3}\right)$ which has been measured by Guillot et al. [6]. The theoretical FIR spectrum was obtained only from an electrostatic induced contribution decomposed in its quadrupole and hexadecapole components,

$$
\alpha(\omega)=\alpha^{(I)}(\omega)=\alpha^{(\Theta)}(\omega)+\alpha^{(\Phi)}(\omega),
$$

where the induced contributions $\alpha^{(\Theta)}(\omega)$ and $\alpha^{(\Phi)}(\omega)$ have been obtained from Eq. (8) with $\lambda=2$ and 4 . We have taken the values $B_{\mathrm{N}_{2}}=1.999$ $\mathrm{cm}^{-1}[33], \alpha_{\mathrm{Xe}}=4.11 \AA^{3}[6]$, and $n=1.257$. Only contributions arising from the anisotropic term $J=2$ of the intermolecular potential (16) are considered. Furthermore, most recent experimental and theoretical values available in the literature for the quadrupole and hexadecapole moments of $\mathrm{N}_{2}$ in its ground vibrational state lie in the ranges:

$$
|\Theta|=[1.2,1.65] \mathrm{D} \AA, \quad|\Phi|=[2.1,4.1] \mathrm{D} \AA^{3} .
$$

We have considered these ranges for a first estimation of the translational strength parameters $C_{2,3}$ and $C_{4,5}$.

The calculation of the translational functions, $\widehat{C}_{t r}^{(\lambda, \lambda+1)}(\omega)$, with $\lambda=2$ and 4 , was carried out from MD simulations. The $\mathrm{N}_{2}$ molecule has been considered as isotropic and the interactions $\mathrm{N}_{2}-\mathrm{Xe}$ and $\mathrm{Xe}-\mathrm{Xe}$ were considered to be of the 12-6 Lennard-Jones type with parameters [6]: $\sigma_{\mathrm{N}_{2}-\mathrm{Xe}}=3.74$ $\AA, \epsilon_{\mathrm{N}_{2}-\mathrm{Xe}}=166.3 \mathrm{~K}$, and $\sigma_{\mathrm{Xe}-\mathrm{Xe}}=3.89 \AA, \epsilon_{\mathrm{Xe}-\mathrm{Xe}}=282.4 \mathrm{~K}$.

The $\lambda$-rotational functions $\widehat{C}_{\text {rot }}^{(\lambda)}(\omega)$, with $\lambda=2$ and 4 , were calculated from Eqs. (18). Therefore, the evaluation of the quadrupolar, $\alpha^{(\Theta)}(\omega)$, and 


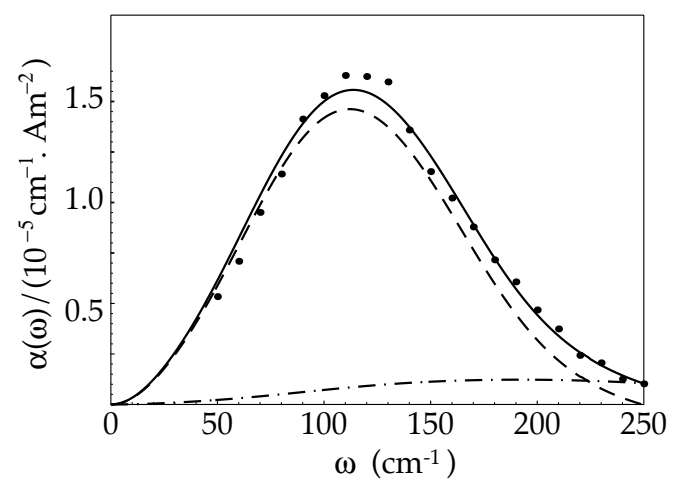

Figure 2. FIR spectrum of a $\mathrm{N}_{2}$-Xe gaseous mixture at $T=295 \mathrm{~K}, \rho_{\mathrm{N}_{2}}=3$ amagat and $\rho_{\mathrm{Xe}}=350$ amagat. Theoretical absorption coefficient obtained by considering the quantum $\lambda$-rotational spectra with $|\Theta|=1.52 \mathrm{D} \AA,|\Phi|=3.22 \mathrm{D}^{3}, \kappa_{2}=58.8 \times 10^{-22} \mathrm{~J}$, and $t_{2}=7.2 \times 10^{-15} \mathrm{~s}$ : total line shape (solid line), quadrupolar contribution $\alpha^{(\Theta)}(\omega)$ (dashed line), and hexadecapolar contribution $\alpha^{(\Phi)}(\omega)$ (dash-dotted line). Experimental spectrum (dots).

the hexadecapolar, $\alpha^{(\Phi)}(\omega)$, contributions to $\alpha(\omega)$ can be performed, for given values of the quadrupole $|\Theta|$ and hexadecapole $|\Phi|$ moments of $\mathrm{N}_{2}$. We have considered the statistical parameters as a set of adjustable parameters with values chosen by comparison with the experimental spectrum. In this fitting procedure we have considered that the main contribution to absorption comes from the quadrupolar induction mechanism, and we have added the hexadecapolar contribution in order to reproduce the high frequency behavior of the experimental spectrum without exceeding its maximum intensity. We have found a satisfactory agreement between the theoretical and experimental spectra for $\kappa_{2} \geq 30 \times 10^{-22} \mathrm{~J}, t_{2} \leq 2.6 \times 10^{-14} \mathrm{~s}$, and values of the quadrupole and hexadecapole moments lying in the ranges:

$$
|\Theta|=[1.47,1.60] \mathrm{D} \AA, \quad|\Phi|=[0,5.20] \mathrm{D}^{3} .
$$

Regarding the quadrupole moment we must underline that the obtained range is contained in the corresponding interval given in (30). However, the interval of values obtained for the hexadecapole is wider than the expressed in (30). In order to attain a more precise estimation for the value of the hexadecapole, we have considered the quadrupole moment as a fixed parameter, and performed the fitting procedure only for the remaining three parameters of the theory, i.e., $|\Phi|, \kappa_{2}$ and $t_{2}$. Assuming the value $|\Theta|=1.52$ DA reported by Dagg et al. [34], we have found the best agreement between 
the theoretical and the experimental spectra for $\kappa_{2}=(60 \pm 20) \times 10^{-22} \mathrm{~J}$, $t_{2}=(7.2 \pm 0.8) \times 10^{-15} \mathrm{~s}$, and $|\Phi|=3.2 \pm 0.3 \mathrm{D}^{3}$.

The calculated spectrum represented in Fig. 2 corresponds to the last case. The good agreement between the experimental and the calculated spectra together with the drastic reduction of the interval of values corresponding to the hexadecapole moment allows to consider this interval as a fairly good estimation of the value of this quantity. The obtained value for the hexadecapole moment is very close to the value $|\Phi|=3 \pm 1 \mathrm{D}^{3}$ reported by Birnbaum and Cohen [33], and greater than the one reported by Maroulis and Thakkar [35] obtained from many-body perturbation theory calculations.

The quadrupolar, $\alpha^{(\Theta)}(\omega)$, and the hexadecapolar, $\alpha^{(\Phi)}(\omega)$, contributions to the calculated spectrum $\alpha(\omega)$ are also sketched in Fig. 2. A glance to this figure allows to corroborate that $\alpha^{(\Theta)}(\omega)$ is the most significant contribution to the absorption intensity, while $\alpha^{(\Phi)}(\omega)$ is revealed as important in order to describe the behavior of the experimental spectrum at high frequencies.

\section{Translational/rotational contributions and static cancellation effects}

The FIR spectra of CO-Ar at high-gas and liquid densities show that the consideration of electric multipolar induction is essential in order to properly reproduce the available experimental results for these systems. The relevant degrees of freedom contributing to induced absorption are the rotation of the diatomic and the translation of the solvent atoms and the diatomic itself. These degrees of freedom are coupled via the anisotropic part of the solute-solvent interaction, but assuming the translational decoupling approximation, the spectra can be expressed as a convolution of a translational part and a rotational one. On the other hand, the translational part can be expressed as a sum of two- and three-body components, which allows one to analyze the possible existence of many-body static cancellation effects in the total integrated absorption coefficient (IAC) and their dependence with the range of the induction mechanism. The study of how the different degrees of freedom of the system affect the existence of cancellation or enhancement effects, and the influence of thermodynamic conditions on these effects have been also object of our interest in the last years.

With these goals in mind we have considered the general expression for the IAC associated to each $\lambda L$ term in expansion (4), $\alpha_{1, \lambda L}$. The mentioned 
expression was firstly derived by Poll and van Kranendonk [36], and reads:

$$
\alpha_{1, \lambda L} \equiv \alpha_{1, \lambda L}^{(r)}+\alpha_{1, \lambda L}^{(t)},
$$

where the indices $(r)$ and $(t)$ stands for rotation and translation respectively. Moreover, it is possible to split each contribution in two-body $(2 b)$ and three-body (3b) terms, $\alpha_{1, \lambda L}^{(r)}=\alpha_{1, \lambda L}^{(r, 2 b)}+\alpha_{1, \lambda L}^{(r, 3 b)}$ and $\alpha_{1, \lambda L}^{(t)}=\alpha_{1, \lambda L}^{(t, 2 b)}+$ $\alpha_{1, \lambda L}^{(t, 3 b)}$ with:

$$
\begin{gathered}
\alpha_{1, \lambda L}^{(r, 2 b)}=\frac{2 \pi^{2} \rho_{A}}{3 c} \frac{\lambda(\lambda+1)}{I_{A}} \sum_{k}<B_{\lambda L}^{2}\left(R_{k}\right)> \\
\alpha_{1, \lambda L}^{(r, 3 b)}=\frac{2 \pi^{2} \rho_{A}}{3 c} \frac{\lambda(\lambda+1)}{I_{A}} \sum_{k \neq k^{\prime}}<B_{\lambda L}\left(R_{k}\right) B_{\lambda L}\left(R_{k^{\prime}}\right) P_{L}\left(\cos \theta_{k, k^{\prime}}\right)> \\
\alpha_{1, \lambda L}^{(t, 2 b)}=\frac{2 \pi^{2} \rho_{A}}{3 c} \sum_{k} \frac{1}{m_{A B}}\left[<\left(\frac{d B_{\lambda L}\left(R_{k}\right)}{d R_{k}}\right)^{2}>+L(L+1)<\frac{B_{\lambda L}^{2}\left(R_{k}\right)}{R_{k}^{2}}>\right] \\
\alpha_{1, \lambda L}^{(t, 3 b)}=\frac{2 \pi^{2} \rho_{A}}{3 c} \frac{1}{m_{A}}\left[\frac{L+1}{2 L+1} \sum_{k \neq k^{\prime}}<C_{\lambda L}\left(R_{k}\right) C_{\lambda L}\left(R_{k^{\prime}}\right) P_{L+1}\left(\cos \theta_{k, k^{\prime}}\right)>+\right. \\
\left.+\frac{L}{2 L+1} \sum_{k \neq k^{\prime}}<D_{\lambda L}\left(R_{k}\right) D_{\lambda L}\left(R_{k^{\prime}}\right) P_{L-1}\left(\cos \theta_{k, k^{\prime}}\right)>\right]
\end{gathered}
$$

In these equations $m_{A B}$ is the solute-solvent reduced mass, $I_{A}$ is the moment of inertia of the diatomic molecule, $m_{A}$ its mass, and the remaining symbols have their usual meaning. Besides, we have defined the functions $C_{\lambda L}\left(R_{k}\right)$ and $D_{\lambda L}\left(R_{k}\right)$ as:

$$
\begin{gathered}
C_{\lambda L}\left(R_{k}\right)=\frac{d B_{\lambda L}\left(R_{k}\right)}{d R_{k}}-L \frac{B_{\lambda L}\left(R_{k}\right)}{R_{k}}, \\
D_{\lambda L}\left(R_{k}\right)=\frac{d B_{\lambda L}\left(R_{k}\right)}{d R_{k}}+(L+1) \frac{B_{\lambda L}\left(R_{k}\right)}{R_{k}} .
\end{gathered}
$$

The details of the calculations and the necessary hypotheses to obtain Eqs. (32)-(36) can be found elsewhere in the literature [27],[36]-[38] and specially in Ref. [9]. We only remark here that, in general, in the FIR spectra of these systems, there exist a non-negligible contribution coming from the 
interference between pure induced and pure allowed spectra. Only when an isotropic diatom-atom potential is assumed, interference terms vanish. One of the assumptions underneath the above equations is precisely the isotropy of the interactions, and thus these expressions allow to calculate only the integrated absorption coefficients of the pure terms contributing to absorption. We restrict our discussion only to those pure induced spectral moments with radial functions $B_{\lambda L}\left(R_{k}\right)$ given by Eq. (4).

The thermodynamic conditions we have elected to perform a systematic study of $\alpha_{1, \lambda L}$ for $\mathrm{CO}$ in liquid $\mathrm{Ar}$ are the following:

(1) Evolution with temperature for a fixed solvent density, $\rho=1.3 \mathrm{~g} / \mathrm{cm}^{3}$, and temperatures between 90 and $150 \mathrm{~K}$. This interval in the temperature approximately covers all the range between the temperature of the triple and critical points for Ar.

(2) Evolution with density for a fixed temperature, $T=130 \mathrm{~K}$ and densities between 1.1 and $1.5 \mathrm{~g} / \mathrm{cm}^{3}$. This density interval roughly covers all the region between the solid-liquid and liquid-gas coexistence curves.

For all these thermodynamic states we performed molecular dynamics simulations with the same technical details that in Sec. 3. The results have been normalized in such a way that they are independent of the solute density and the values of the electric multipole moments of CO. This normalization reads:

$$
\bar{\alpha}_{1, \lambda L} \equiv \frac{1}{4 \pi \rho_{B}} \frac{\alpha_{1, \lambda L}}{\alpha_{1, \mu_{\mathrm{p}}}} \frac{\mu_{\mathrm{p}}^{2}}{Q_{\lambda}^{2}},
$$

where $\alpha_{1, \mu_{\mathrm{p}}}=\rho_{A}\left(2 \pi \mu_{\mathrm{p}}^{2}\right) /\left(3 c I_{A}\right)$ is the integrated permanent dipole absorption.

\subsection{EVOLUTION WITH TEMPERATURE AND DENSITY}

The evolution with temperature of the translational, $\bar{\alpha}_{1, \lambda L}^{(t)}$, and, rotational, $\bar{\alpha}_{1, \lambda L}^{(r)}$, contributions is displayed in Fig. 3. For dipole induced-dipole $(\lambda=$ 1 ), both contributions are very similar in magnitude, but when we go to higher multipolar terms the rotational contribution is always clearly greater than the translational one for any temperature. This can be understood in terms of the range of the multipolar induced mechanisms: higher multipolar terms correspond to shorter ranges and at short ranges translation is more hindered than rotation. Respect to the relative evolution with $T$, both contributions increase almost linearly with temperature, and $\bar{\alpha}_{1, \lambda L}^{(r)}$ always increase with $T$ clearly faster than $\bar{\alpha}_{1, \lambda L}^{(t)}$.

The evolution with density of the translational, $\bar{\alpha}_{1, \lambda L}^{(t)}$, and, rotational, $\bar{\alpha}_{1, \lambda L}^{(r)}$, contributions is displayed in Fig. 4. The translational component 

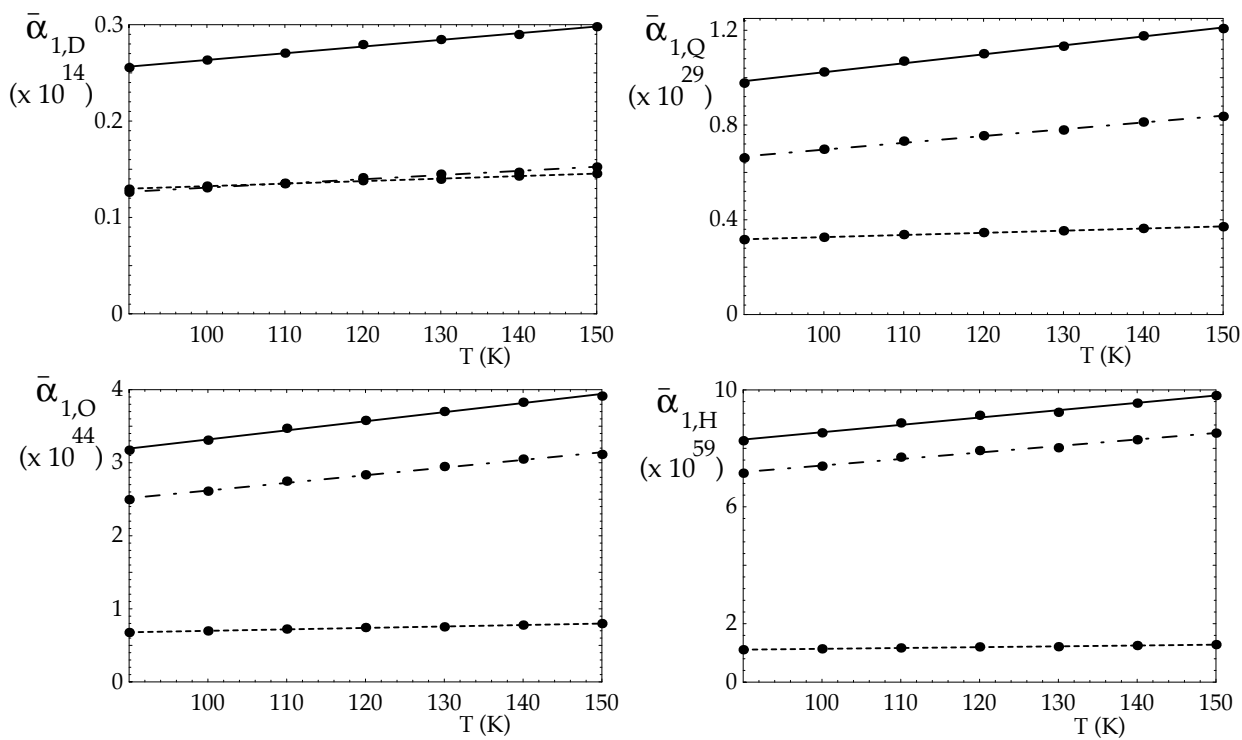

Figure 3. Evolution with temperature of the translational, $\bar{\alpha}_{1, \lambda L}^{(t)}$, and rotational, $\bar{\alpha}_{1, \lambda L}^{(r)}$, components of $\bar{\alpha}_{1, \lambda L}$. Lines represent linear fittings for each term: solid line, $\bar{\alpha}_{1, \lambda L}$; dashed line, $\bar{\alpha}_{1, \lambda L}^{(t)}$; and lines-points, $\bar{\alpha}_{1, \lambda L}^{(r)}$. Units are $\mathrm{cm}^{3-2 \lambda} / \mathrm{g}$ for each multipolar order.
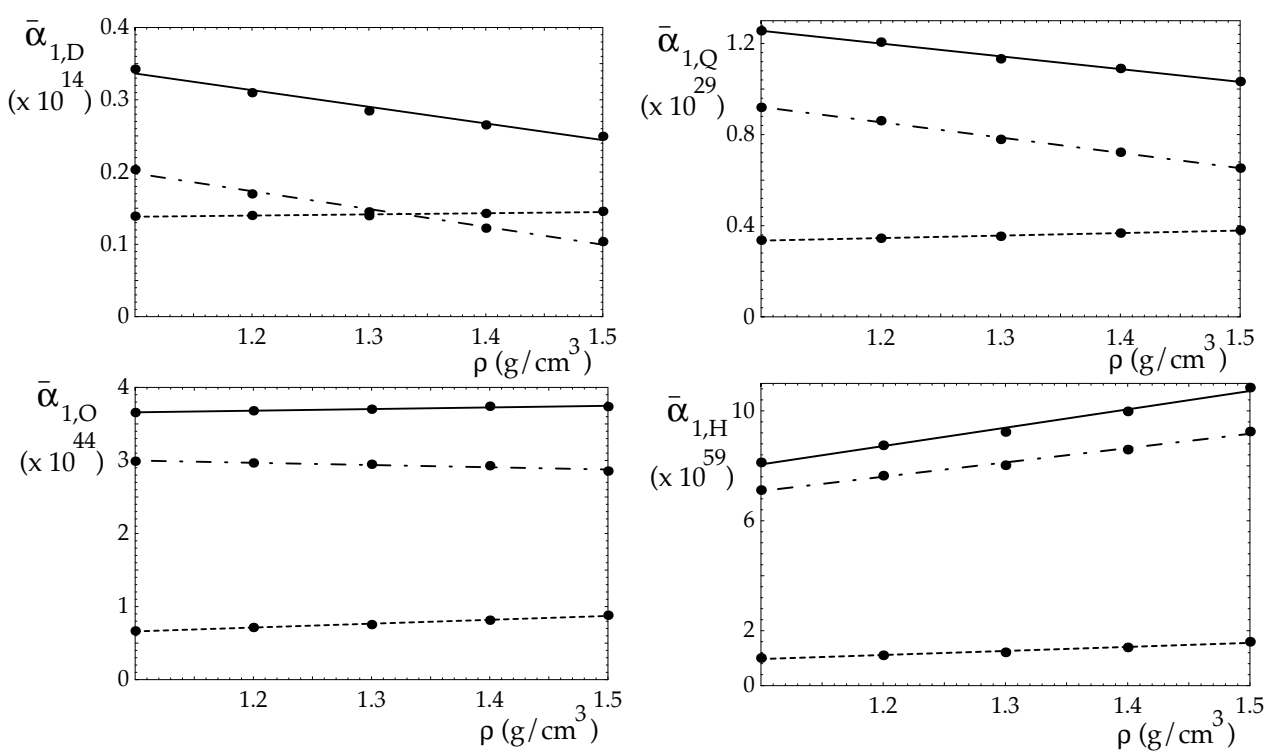

Figure 4. Evolution with the solvent density of the translational and rotational components of $\bar{\alpha}_{1, \lambda L}$. Notation and units are as in Fig. 3 . 
of $\bar{\alpha}_{1, \lambda L}$ always increase linearly with density. This increase is greater for higher multipolar orders. Nevertheless, the density behavior of $\bar{\alpha}_{1, \lambda L}^{(r)}$ is more complicated. For $D, Q$ and $O$, it decreases with density (decreasing that diminishes with the multipolar order), but for $H$ it increases with density. In any case the density behavior of $\bar{\alpha}_{1, \lambda L}^{(t)}$ is smoother than that of $\bar{\alpha}_{1, \lambda L}^{(r)}$ and this causes that the density evolution of the total integrated coefficient $\bar{\alpha}_{1, \lambda L}$ is mainly associated to $\bar{\alpha}_{1, \lambda L}^{(r)}$. With respect to magnitudes, $\bar{\alpha}_{1,12}^{(r)}$ and $\bar{\alpha}_{1,12}^{(t)}$ are similar, with $\bar{\alpha}_{1,12}^{(r)}>\bar{\alpha}_{1,12}^{(r)}$ for low densities and the opposite for high densities. For all other multipolar terms, the rotational component is always greater than the translational one.

Then, as a first conclusion, we obtain that for the CO-Ar system, the evolution of the pure induced components of the IAC with the thermodynamic conditions of the solvent is essentially due to the evolution of the rotation of the diatomic, specially for high-order electric multipolar terms.

\subsection{CANCELLATION EFFECTS AND DEGREES OF FREEDOM}

We have also evaluated from MD simulations the two- and three-body components of $\bar{\alpha}_{1, \lambda L}$. The obtained results are reported in Table 2 . These results show that the sign of the three-body components (i.e., the existence of destructive or constructive interferences) does not change in all the liquid phase, and so, the existence of cancellation or enhancement effects is independent of temperature or density. It seems to be only affected by the multipolar order, that is, the range of the functions to be averaged to compute $\bar{\alpha}_{1, \lambda L}$. Cancellation effects for long-range electric multipolar induction $(D, Q$, and $O)$ progressively decrease and even get into enhancement when the range is low enough (for $H$ ). Furthermore, our results also show that cancellation effects for $D, Q$ and $O$ are more complete (the absolute values of two- and three-body terms are more similar) as the isotropy of the fluid increase, that is, cancellation increases as density increases and temperature decreases.

From the results of Table 2 one can also conclude that the translational components present cancellation effects for dipole induced-dipole absorption and quadrupole induced-dipole absorption. For octupole and hexadecapole this cancellation moves to enhancement (notice that in the total integrated coefficient there exist enhancement only for hexadecapole). Moreover, the existence of cancellation or enhancement for the translational component seems to be independent of the thermodynamic state in the range we are considering. The thermodynamic conditions seems to be important only with respect to the completeness of cancellation or enhancement. For the rotational component the situation is slightly different, because there 


\begin{tabular}{|c|c|c|c|c|c|c|c|c|}
\hline \multirow[b]{2}{*}{$T(\mathrm{~K})$} & \multicolumn{4}{|c|}{$\bar{\alpha}_{1, \lambda L}^{(t, 3 b)} / \bar{\alpha}_{1, \lambda L}^{(t, 2 b)}$} & \multicolumn{4}{|c|}{$\bar{\alpha}_{1, \lambda L}^{(r, 3 b)} / \bar{\alpha}_{1, \lambda L}^{(r, 2 b)}$} \\
\hline & $\begin{array}{c}D \\
\left(R_{k}^{-8}\right)\end{array}$ & $\begin{array}{c}Q \\
\left(R_{k}^{-10}\right)\end{array}$ & $\begin{array}{c}O \\
\left(R_{k}^{-12}\right)\end{array}$ & $\begin{array}{c}H \\
\left(R_{k}^{-14}\right)\end{array}$ & $\begin{array}{c}D \\
\left(R_{k}^{-6}\right)\end{array}$ & $\begin{array}{c}Q \\
\left(R_{k}^{-8}\right)\end{array}$ & $\begin{array}{c}O \\
\left(R_{k}^{-10}\right)\end{array}$ & $\begin{array}{c}H \\
\left(R_{k}^{-12}\right)\end{array}$ \\
\hline 90 & -0.44 & -0.27 & 0.02 & 0.25 & -0.87 & -0.74 & -0.46 & 0.04 \\
\hline 120 & -0.42 & -0.25 & 0.03 & 0.21 & -0.85 & -0.72 & -0.43 & 0.06 \\
\hline 150 & -0.41 & -0.23 & 0.04 & 0.19 & -0.84 & -0.70 & -0.40 & 0.06 \\
\hline$\rho\left(\mathrm{g} / \mathrm{cm}^{3}\right)$ & $D$ & $Q$ & $O$ & $H$ & $D$ & $Q$ & $O$ & $H$ \\
\hline 1.1 & -0.37 & -0.19 & 0.04 & 0.15 & -0.78 & -0.63 & -0.33 & 0.06 \\
\hline 1.3 & -0.41 & -0.24 & 0.03 & 0.20 & -0.85 & -0.71 & -0.41 & 0.06 \\
\hline 1.5 & -0.46 & -0.30 & 0.01 & 0.29 & -0.90 & -0.78 & -0.51 & 0.02 \\
\hline
\end{tabular}

TABLE 2. Magnitude of cancellation effects for the translational and rotational components of $\bar{\alpha}_{1, \lambda L}$ for the system $C O-A r$ at different temperatures and densities. The upper part of the Table corresponds to a systematic study with temperature for a fixed density $\left(1.3 \mathrm{~g} / \mathrm{cm}^{3}\right)$ and the lower part to a systematic study with density for a fixed temperature $(130 \mathrm{~K})$. Under the symbol of each contribution its radial decay is displayed. Note that for any component cancellation is absent when the decay is equal or faster than $R_{k}^{-12}$.

exist constructive interference between many-body terms only for hexadecapole. In any other case and for any temperature or density, cancellation is clear. We also note that cancellation (when it exists) is always greater in rotational components than in translational ones.

\section{Concluding remarks}

In the last years we have devoted special attention to the study of FIR absorption spectra of both polar diatomic molecules with a low enough permanent dipole moment and nonpolar diatomic molecules in nonpolar fluids. In this context, our studies were focused on two main aspects.

First, FIR spectra give valuable information about the nature of the induction processes arising from the diatom-atom interactions. In the case of a very diluted solution at low and moderate gas densities and a highly polarizable solvent, the main induced contributions to the absorption intensity come from the multipole-induced dipole mechanisms. Hence, this type of spectra can be useful to obtain values of the leading multipole moments of the diatomic molecule. This is the case of $\mathrm{CO}-\mathrm{Ar}$ and $\mathrm{N}_{2}$-Xe solutions, for which experimental FIR spectra at different densities are available. We have analyzed theoretically these spectra in order to estimate the leading multipoles of $\mathrm{CO}$ and $\mathrm{N}_{2}$. The theoretical line shapes were obtained from the convolution of translational and rotational components. The former have been derived both from classical approachs and MD simulations, 
while the latter have been evaluated by assuming that the translational degrees of freedom behave as a thermal bath and the rotational dynamics of the diatomic is described by means of a quantum rigid rotor stochastically interacting with the bath.

Second, we have shown the existence, at dense-gas and liquid densities, of static cancellation effects between many-body components of the pure induced terms of the FIR integrated absorption coefficient. These cancellation effects decrease when going to high multipolar induction mechanisms, and even for hexadecapole induced-dipole cancellation disappears and transforms into enhancement. We have been interested in the influence of solvent temperature and density in the completeness of these effects and we have also tried to elucidate how the active degrees of freedom of the system affect the existence of cancellation or enhancement effects. In particular, for COAr at liquid densities, we concluded that when it exists, cancellation is more complete in these conditions where the liquid is more isotropic: low temperatures and high densities. Furthermore, the magnitude of cancellation is always more complete in rotational components. Moreover in any case rotational components are more important than translational ones, except for the dipole induced-dipole term, in which the importance of rotation and translation is similar.

Also, we would like to stress the following points:

(i) A crucial assumption in our spectral theory is the translational decoupling approximation. It is a bit difficult to assess to what extent our estimates of the multipole moments of $\mathrm{CO}$ and $\mathrm{N}_{2}$ depend on this approximation. In the low-gas density case, the excellent agreement between the value (25) and the measured by Meerts et al. [29] for the quadrupole moment of CO seems to justify a posteriori such approximation. In the liquid case, the small density fluctuations make more consistent the decoupling approximation and thus one can hope that it does not practically influence on the corresponding estimates. Dense gas cases are subtler. Only comparisons with MD calculations seem to be the way of checking this approximation. In any case, we would like to remark that the translational decoupling picture works properly in order to reproduce all the considered spectra.

(ii) Our spectral theory only considers the linear isotropic polarizability of the solvent, $\alpha_{B}$. Our estimates for $Q_{\lambda}$ have been made for a fixed value of $\alpha_{B}$. Any change in its numerical value implies a modification of $Q_{\lambda}$ values, so that $\alpha_{B} \cdot Q_{\lambda}=$ cte in each considered case. In this context, it would be of interest to notice that interatomic interactions can induce an anisotropic polarizability. We have checked [37] that for the CO-Ar mixture at lowgas density anisotropic polarizability correction is negligible. As density increases, one can also expect that the anisotropic polarizability of the 
solvent takes a very small value compared to the corresponding isotropic polarizability due to many-body cancellation effects.

(iii) Contribution due to the higher order electric multipoles was found to appreciably increase with density. This interesting effect, tentatively attributed to possible influence of the irreducible three-body dipoles or of the short-ranged (overlap) induction, certainly deserves further study.

(iv) We have assumed that only electrostatic long-ranged induction mechanisms are responsible for the FIR absorption in the systems under study. Other contributions due to back induction, field-gradient effects, and nonzero second order (fourth rank tensor) hyperpolarizability of the solvent molecules are not considered. Ordinarily these effects can be neglected. Recently Samios et al. [39] calculated back-induction contributions in the infrared spectra of $\mathrm{HCl}$ in $\mathrm{CCl}_{4}$.

(v) Interference contributions arising from permanent-induced and induced-induced cross-correlations have not been considered. Classical MD simulations [40] for CO-Ar show that at high-gas and liquid densities these interferences can reach a $3 \%$ of the total FIR integrated absorption. Unfortunately, a quantum spectral theory taken into account these interference effects is yet not available.

Finally, we feel important to mention that in the last years interesting spectral theories [41] capable to take into account interferences among absorption lines and among spectral branches at any order have been proposed. These theories improve the secular-interference scheme by a new one based upon diagonal and non-diagonal terms, in which interferences can be calculated in an exact way. Moreover, they are formulated independently of the spectral region considered and were succesfully applied to far- and near-infrared, and Raman absorption [42]. It would be a challenging work in the future to extend this formalism to study the importance of interaction-induced effects in some open problems, as the appearance of Q-branch absorption on the central range of the near-infrared bands of hydrogen halides in simple liquid solvents [43].

\section{Acknowledgments}

We would like to thank Prof. M.O. Bulanin for many helpful and elucidating discussions. We thank financial support from the Ministerio de Ciencia y Tecnología of Spain under Grant BFM 2002-01225 FEDER, and by Junta de Castilla y León under Grant SA097/01.

\section{References}

1. M. O. Bulanin,S. Velasco, and A. Calvo Hernández, J. Mol. Liq. 70, 107 (1996).

2. A. Calvo Hernández, S. Velasco, and F. Mauricio, Phys. Rev. A 31, 3419 (1985). 
3. J. M. M. Roco, A. Medina, A. Calvo Hernández, and S. Velasco, Chem. Phys. Lett. 216, 593 (1993).

4. U. Buontempo, S. Cunsolo, and G. Jacucci, J. Chem. Phys. 59, 3750 (1973).

5. U. Buontempo, S. Cunsolo, and G. Jacucci, Mol. Phys. 21, 381 (1971).

6. B. Guillot, Ph. Marteau, and J. Obriot, Mol. Phys. 65, 765 (1988).

7. G. Birnbaum and B. Guillot in 'Spectral Line Shapes', edited by M. Zoppi and L. Ulivi (Firenze, Italy, 1996).

8. M. O. Bulanin, Opt. Spectrosc. 72, 45 (1992).

9. J. M. M. Roco, A. Medina, A. Calvo Hernández and S. Velasco, J. Chem. Phys. 107, 4844 (1997).

10. A. Medina, J. M. M. Roco, A. Calvo Hernández and S. Velasco, J. Chem. Phys. 108, 9480 (1998).

11. R. G. Gordon, Adv. Magn. Reson. 3, 1 (1968).

12. V. V. Bertsev, in Molecular Cryospectroscopy, edited by R. J. H. Clark and R. H. Hester (John Wiley \& Sons, 1995), pp 1-19.

13. J. D. Poll and J. L. Hunt, Can. J. Phys. 54, 461 (1976); G. Birnbaum, G. Bachet, and L. Frommhold, Phys. Rev. A 36, 3729 (1987).

14. J. D. Poll and J. Van Kranendonk, Can. J. Phys. 39, 189 (1961).

15. J. D. Poll and J. L. Hunt, Can. J. Phys. 54, 461 (1976).

16. G. Birnbaum, G. Bachet, and L. Frommhold, Phys. Rev. A 36, 3729 (1987).

17. D. Frenkel, PhD. Thesis. University of Amsterdam (1977).

18. A. Borysow and M. Moraldi, J. Chem. Phys., 99, 8424 (1993).

19. H. Mori, Prog. Theor. Phys. 33, 423 (1965); 34, 399 (1965).

20. U. Balucani, V. Tognetti, and R. Vallauri, Phys. Rev. A 19, 177 (1979).

21. B. Guillot and G. Birnbaum, J. Chem. Phys. 79, 686 (1983).

22. J. G. Kirkwood, J. Chem. Phys. 3, 300 (1935).

23. Ph. Marteau, J. Obriot, F. Fonder and B. Guillot, Mol. Phys. 59, 1305 (1986).

24. J. Chesnoy, Chem. Phys. 83, 283 (1984).

25. W. A. Herrebout, B. J. Van der Veken, A. Medina, A. Calvo Hernández, and M. O. Bulanin, Mol. Phys. 96, 1115-1124 (1999).

26. J. M. M. Roco, A. Medina, A. Calvo Hernández and S. Velasco, J. Chem. Phys. 103, 9161-9174 (1995).

27. A. Medina, S. Velasco, and A. Calvo Hernández, Phys. Rev., A44, 3023 (1991).

28. A. Medina, S. Velasco, and A. Calvo Hernández, Phys. Rev., A45, 5289 (1992).

29. W. L. Meerts, F. H. Leeuw and A. Dynamus, Chem. Phys. 22, 319 (1977).

30. G. H. F. Diercksen and A. J. Sadlej, Chem. Phys. 96, 17 (1985).

31. D. G. Bounds and S. Wilson, Mol. Phys. 54, 445 (1985).

32. R. M. van Aalst and J. van der Elsken, Chem. Phys. Lett. 23, 198 (1973).

33. G. Birnbaum and E. R. Cohen, Mol. Phys. 32, 161 (1976).

34. I. R. Dagg, A. Anderson, S. Yang, W. Smith, and L. A. A. Read, Can. J. Phys. 63, 625 (1985).

35. G. Maroulis and A. J. Thakkar, J. Chem. Phys. 88, 7623 (1988).

36. J. D. Poll and J. van Kranendonk, Can. J. Phys. 39, 189 (1961).

37. J. M. M. Roco, A. Medina, A. Calvo Hernández and S. Velasco, J. Chem. Phys. 103, 9175 (1995).

38. L. Frommhold, 'Collision-Induced Absorption in Gases' (Cambridge University Press, Cambridge, 1993) Chap. 6.

39. G. Chatzis, and J. Samios, J. Phys. Chem., A105, 9522 (2002).

40. A. Medina, A. Calvo Hernández, J. M. M. Roco, and S. Velasco, J. Mol. Liq., 70, 169 (1996).

41. A. Padilla, J. Pérez, and A. Calvo Hernández, J. Chem. Phys. 111, 11015 (1999).

42. A. Padilla, J. Pérez, and A. Calvo Hernández, J. Chem. Phys. 111, 11026 (1999); 113, 4290 (2000).

43. A. Medina, J. M. M. Roco, A. Calvo Hernández, S. Velasco, M. O. Bulanin, W. A. Herrebout and B. J. van der Veken, J. Chem. Phys. 116, 5058 (2002). 